\title{
Photonic High-Bandwidth RF Splitter With Arbitrary Amplitude and Phase Offset
}

\author{
Leimeng Zhuang, Bill Corcoran, Chen Zhu, and Arthur J. Lowery, Fellow, IEEE
}

\begin{abstract}
This letter presents an alternative approach to achieve a high-bandwidth RF splitter functionality based on a microwave photonic system. The proposed approach has an arbitrary amplitude and a phase offset between the two outputs. In addition, it features a simple system architecture and easy tuning mechanism. In the experimental demonstration, an instantaneous RF band from 5 to $20 \mathrm{GHz}$ was shown.
\end{abstract}

Index Terms-Microwave photonics, RF coupler, phase shifter, arbitrary filter coefficient, FIR.

\section{INTRODUCTION}

$\mathbf{M}$ ICROWAVE photonics (MWP) has been stimulating a revolution in RF engineering by incorporating photonic components or subsystems which enable unprecedented RF functionality and features [1]. MWP enjoys the advantages inherent to photonics, such as large instantaneous bandwidth, easy implementation of tuning, reduced electromagnetic interference, small size, low weight, low power consumption, and low cost potentials. Driven by the considerable interest from both academic and industrial community, a large number of MWP investigations have been conducted with respect to RF generation, transmission, processing, control, and instrumentation [1], [2].

In line with this effort, we propose and experimentally demonstrate an alternative MWP approach to achieve arbitrary $1 \times 2$ splitting of an RF signal, where arbitrary amplitude and phase offset between the two outputs can be introduced over a multi-octave instantaneous bandwidth. These desirable features can be utilized in some interesting RF applications where two copies of a broadband signal with tunable phase and/or amplitude offset are required, such as flexible analog encryption in RF transmission systems [3], complex signal detection in radar systems [4], and generation of arbitrary radiation polarization for dual-linear transmit antennas [5]. To our best knowledge, such arbitrary RF splitter functionality is not yet available in a single packaged device. Although bulky systems constructed using discrete RF power splitters, tunable phase shifters, and tunable attenuators may be

Manuscript received June 4, 2014; revised August 7, 2014; accepted August 13, 2014. Date of publication August 18, 2014; date of current version September 30, 2014. The work of A. J. Lowery was supported by the Australian Research Committee Laureate Fellowship under Grant FL130100041 within the framework of the Electro-Photonic Interchange Project.

The authors are with the Electro-Photonic Interchange Laboratory, Department of Electrical and Computer Systems Engineering, Monash University, Clayton, VIC 3800, Australia (e-mail: leimeng.zhuang@ monash.edu; bill.corcoran@monash.edu; chen.zhu@monash.edu; arthur. lowery@monash.edu).

Color versions of one or more of the figures in this letter are available online at http://ieeexplore.ieee.org.

Digital Object Identifier 10.1109/LPT.2014.2349010 used as an all-electronics solution, it is very difficult, if not impossible, to achieve a multi-octave instantaneous bandwidth and large-range tunability simultaneously. Alternatively, MWP techniques offer simple approaches to realize such broadband RF splitter functionality with easy tuning and potentials of full-system integration. Previous studies have identified MWP approaches for introducing arbitrary RF phase shift over a wide instantaneous bandwidth, using various optical devices, such as an optical spectrum processor [6], gratings [7], [8], semiconductor optical amplifiers [9], [10], waveguide ring resonators [11], [12], polarization modulators [13], [14], and fiber with stimulated Brillouin scattering [15], [16]. The above approaches could provide two outputs using duplication of their circuits, and may not provide independent controls of RF amplitude and phase. The approaches based on photonic integrated circuit building blocks, such as waveguide ring resonators, are favorable in terms of system integration and function scalability. However, it is worth noticing that when realizing a complex system consisting of a large number of building blocks and tuning elements. The control therewith may become an issue. To address this, a dedicated control algorithm needs to be devised, which can be implemented by means of real-time computing or switching between different statuses of a look-up table.

In this letter we present an alternative MWP approach for $1 \times 2$ arbitrary RF splitter functionality with low complexity and easy tuning. In the optical domain, two CW lights with different wavelengths are used to carry the RF signal and produce two copies of it. The core processing comprises of separate carrier phase tuning [11] and spectrum filtering, which allow for independent and arbitrary RF phase and amplitude offset. To implement this, we use a simple combination of a dualparallel Mach-Zehnder modulator (DPMZM) and a demultiplexer, where the device properties enable us to manipulate the phase and amplitude of the spectrum components freely, and the tuning only requires varying optical phase shift in the waveguide being controlled by DC voltages. This approach to realize $1 \times 2$ arbitrary RF splitter is not only advantageous regarding system construction but also promising to become a single packaged device using chip-level integration, as all of the constituent components can be realized as waveguide devices. Therefore, the proposed system is of high interest in terms of system viability, stability, and low-cost potentials.

\section{SYSTEM PRINCIPLE}

\section{A. Signal Processing Scheme}

An illustration of equivalent system functionality is given in Fig. 1, where Fig. 1a shows an arbitrary RF splitter and 


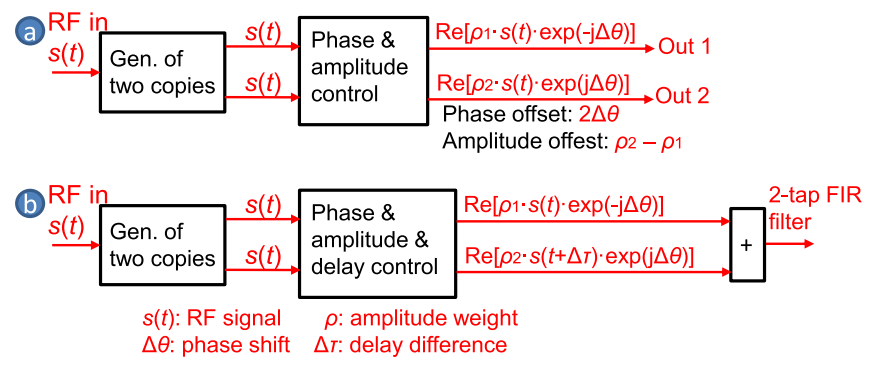

Fig. 1. Illustration of equivalent system functionality: (a) an arbitrary RF splitter and (b) an extended version providing filter functionality.

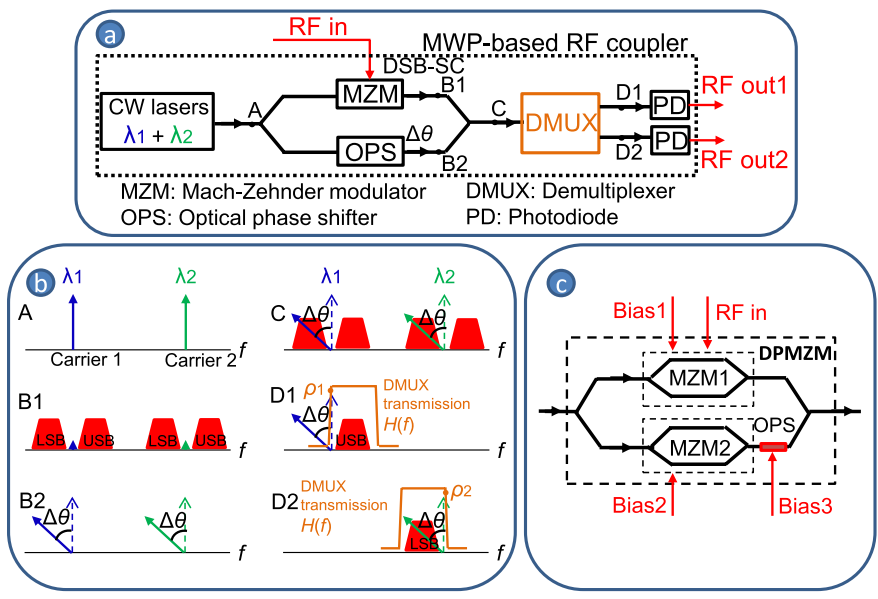

Fig. 2. (a) Schematic of the proposed MWP system to achieve arbitrary RF splitting; (b) spectra illustrating the signals at different points in the system; (c) circuit structure and control scheme of the dual-parallel Mach-Zehnder modulator.

Fig. $1 \mathrm{~b}$ is an extended version providing filter functionality. The major processing includes the generation of two copies of the RF input signal and the control of phase, amplitude, and optionally delay offset between them. For the basic RF splitter functionality, the architecture and working principle of the MWP system is depicted in Fig. 2. Two CW lasers, $\lambda_{1}$ and $\lambda_{2}$ working as two optical carriers, are first fed together into a coupler and then split equally into two separate optical paths. In one path, an input RF signal is impressed on both optical carriers as double-sideband suppressed-carrier (DSB-SC) modulation. In the other path, a common optical phase shift $\Delta \theta$ is applied to both optical carriers. The resulting optical signal spectra are shown in the insets B1 and B2 of Fig. 2b. Here, we use the small-signalmodulation condition where only the $1^{\text {st }}$-order sidebands are considered. Subsequently, the optical signals in these two paths are coupled together again forming a spectrum similar to that of double-sideband full-carrier (DSB-FC) modulation, which is shown in the inset $\mathrm{C}$ of Fig. 2b. Unlike DSB-FC modulation, however, this approach allows for independent tuning of each carrier's phase with respect to the sidebands. In practice, the system section between point $\mathrm{A}$ and $\mathrm{C}$ can be simply implemented using a dual-parallel Mach-Zehnder modulator (DPMZM) [17], a schematic of which is depicted in Fig. 2c. Next, the combined optical signal is sent to a $1 \times 2$ demultiplexer, whose desired transmission characteristics are illustrated in the insets D1 and D2 of Fig. 2b. Here, the demultiplexer plays three roles. One is to demultiplex the optical signals on the two carriers; the second is to suppress one of the two sidebands around each carrier where one carrier loses the opposite sideband to the other; and the last is to introduce amplitude weights to the two carriers, $\rho_{1}$ and $\rho_{2}$ as in Fig. 2b. In practice, the demultiplexer can be implemented using a dedicated waveguide filter, for example an Mach-Zehnder interferometer assisted by ring resonators [18] or an arrayed waveguide grating [19]. According to the system requirements, one can design the filter frequency-domain transmission characteristic $H(f)$ to feature flat passbands with symmetrical transition bands on both sides and flat group delays (for example, Chebyshev Type II filters), and incorporate tuning mechanism to enable frequency shifting of the filter. This will facilitate precise control of $\rho_{1}$ and $\rho_{2}$, and also allow us to compensate for the possible filter frequency drift caused by ambient changes during operation. Besides, it is also possible to make independent changes of $\rho_{1}$ and $\rho_{2}$ by means of power control of the two optical carriers. After detection, the two RF outputs will be two copies of the RF input, and notably output 1 will have an amplitude weight offset of $\Delta \rho_{\mathrm{RF}}=\rho_{1}-\rho_{2}$ and a phase offset of $\Delta \varphi_{\mathrm{RF}}=-2 \Delta \theta$ with respect to output 2 . The cause of this phase offset is that output 1 results from the beating between an optical carrier and its upper sideband (as shown by D1 in Fig. 2b) where the applied carrier phase shift $\Delta \theta$ translates to an additional RF phase shift of $-\Delta \theta$ in the beat product; while output 2 is the opposite case having an additional RF phase shift of $\Delta \theta$, because the beating components are replaced by an optical carrier and its lower sideband (as shown by D2 in Fig. 2b). This effect will also be expressed in the derivations in the following section.

\section{B. Mathematical Description}

Under the condition of small signal modulation where only the $1^{\text {st }}$-order sidebands are considered, the optical signals at points B1, B2, C, D1 and D2 in Fig. 2a can be expressed by

$$
\begin{aligned}
E_{\mathrm{B} 1}= & E_{\mathrm{o}} m(t)\left(\mathrm{e}^{-\mathrm{j}\left(\omega_{\lambda 1} t-\omega_{\mathrm{RF}} t\right)}+\mathrm{e}^{-\mathrm{j}\left(\omega_{\lambda 1} t+\omega_{\mathrm{RF}} t\right)}\right. \\
& \left.+\mathrm{e}^{-\mathrm{j}\left(\omega_{\lambda 2} t-\omega_{\mathrm{RF}} t\right)}+\mathrm{e}^{-\mathrm{j}\left(\omega_{\lambda 2} t+\omega_{\mathrm{RF}} t\right)}\right) \\
E_{\mathrm{B} 2}= & E_{\mathrm{o}}\left(\mathrm{e}^{-\mathrm{j}\left(\omega_{\lambda 1} t+\Delta \theta\right)}+\mathrm{e}^{-\mathrm{j}\left(\omega_{\lambda 2} t+\Delta \theta\right)}\right) \\
E_{\mathrm{C}}= & E_{\mathrm{B} 1}+E_{\mathrm{B} 2}=E_{\mathrm{o}}\left(\mathrm{e}^{-\mathrm{j}\left(\omega_{\lambda 1} t+\Delta \theta\right)}+m(t) \mathrm{e}^{-\mathrm{j}\left(\omega_{\lambda 1} t-\omega_{\mathrm{RF}} t\right)}\right. \\
& +m(t) \mathrm{e}^{-\mathrm{j}\left(\omega_{\lambda 1} t+\omega_{\mathrm{RF}} t\right)}+\mathrm{e}^{-\mathrm{j}\left(\omega_{\lambda 2} t+\Delta \theta\right)} \\
& \left.+m(t) \mathrm{e}^{-\mathrm{j}\left(\omega_{\lambda 2} t-\omega_{\mathrm{RF}} t\right)}+m(t) \mathrm{e}^{-\mathrm{j}\left(\omega_{\lambda 2} t+\omega_{\mathrm{RF}} t\right)}\right) \\
E_{D 1}= & E_{\mathrm{o}}\left(\rho_{1} \mathrm{e}^{-\mathrm{j}\left(\omega_{\lambda 1} t+\Delta \theta\right)}+m(t) \mathrm{e}^{-\mathrm{j}\left(\omega_{\lambda 1} t+\omega_{\mathrm{RF}} t\right)}\right) \\
E_{D 2}= & E_{\mathrm{o}}\left(\rho_{2} \mathrm{e}^{-\mathrm{j}\left(\omega_{\lambda 2} t+\Delta \theta\right)}+m(t) \mathrm{e}^{-\mathrm{j}\left(\omega_{\lambda 2} t-\omega_{\mathrm{RF}} t\right)}\right)
\end{aligned}
$$

where $\omega_{\lambda 1}$ and $\omega_{\lambda 2}$ indicate the frequencies of two optical carriers, $m(t)$ and $\omega_{\mathrm{RF}}$ are the complex amplitude and center frequency of the input RF signal $s(t)=m(t) \cos \left(\omega_{\mathrm{RF}} t\right)$, and $E_{\mathrm{o}}$ represents a reference optical amplitude including the losses in the signal paths. For the sake of simplicity, we ignored the initial phases of the optical carriers and the input RF signal, and assumed that the two optical carriers have equal powers when launched into the system. Then the two system outputs in terms of photocurrent can be derived 
from (4) and (5), giving

$$
\begin{aligned}
& I_{\text {out } 1}=R_{\mathrm{pd}} \cdot\left|E_{\mathrm{D} 1}\right|^{2}=\eta m(t) \cos \left(\omega_{\mathrm{RF}} t-\Delta \theta\right), \\
& I_{\text {out } 2}=R_{\mathrm{pd}} \cdot\left|E_{\mathrm{D} 2}\right|^{2}=\left(1-\Delta \rho_{\mathrm{RF}}\right) \eta m(t) \cos \left(\omega_{\mathrm{RF}} t+\Delta \theta\right),
\end{aligned}
$$

where $R_{\mathrm{pd}}$ is the photodetector responsivity and $\eta$ represents a general RF amplitude factor determined by $E_{0}, R_{\mathrm{pd}}$, and $\rho_{1}$.

\section{EXPERIMENTAl DEMONSTRATION}

\section{A. Laboratory Setup}

To verify the viability of the proposed MWP system for the arbitrary RF splitter, an experimental demonstration was performed using a laboratory setup constructed as in Fig. 2a. The two optical carriers are generated by two tunable $\mathrm{CW}$ lasers operating at $\lambda_{1}=193.1 \mathrm{THz}$ and $\lambda_{2}=193.2 \mathrm{THz}$, with equal powers. The system section between point $\mathrm{A}$ and $\mathrm{C}$ was implemented using a DPMZM (Sumitomo 40G DQPSK). As shown in Fig. 2c, MZM1 was driven by a probe RF signal generated by a vector network analyzer (Anritsu 37247D) and was DC-biased (using Bias 1) at minimum transmission to achieve DSB-SC modulation. In the lower path, MZM2 and the separate optical phase shifter were controlled (using Bias 2 and Bias 3) to vary the carrier amplitude and phase, respectively. For the proof-of-concept purpose, the demultiplexer required in the system was implemented using a programmable optical filter (Finisar Waveshaper 4000S). Its outputs were detected (using an RF photodiode Discovery Semiconductor DSC40S) and the resulting RF outputs were fed to the receive port of the vector network analyzer, where the amplitude and phase offset relative to the RF input can be determined by means of $\mathrm{S}_{21}$ measurement.

\section{B. Measurements on RF Splitter Functionality}

To show the proper system configuration as in Fig. 2b, the optical signal spectrum measurements are presented in Fig. 3. Figure 3a depicts the signal spectrum measured at the output of DPMZM when both MZM1 and MZM2 are biased to the minimum transmission and a driving RF tone with a frequency of $10 \mathrm{GHz}$ is applied to MZM1. Here, only the spectrum around one of the two optical carriers is shown for simplicity, and the achievement of DSB-SC modulation in the upper path of the DPMZM is demonstrated. With MZM1 unchanged and MZM2 biased to the maximum transmission, a spectrum combining the signals from both the upper and lower paths of the DPMZM is achieved (Inset C of Fig. 2b). A measurement of this is shown in Fig. 3b. Figure $3 c$ and $3 d$ show the signal spectra for different RF frequencies, which were measured at the optical filter output, when the filter was configured to simulate a demultiplexer with transmission characteristics as indicated in red dashed lines. In either case, the filter output contains only one of the two optical carriers and one of its two sidebands, where the selection of the remaining sideband agrees with the signal processing scheme in Fig. 2b.

With the system properly configured, the RF signals on the two demultiplexed optical carriers, $\lambda_{1}$ and $\lambda_{2}$, were detected,
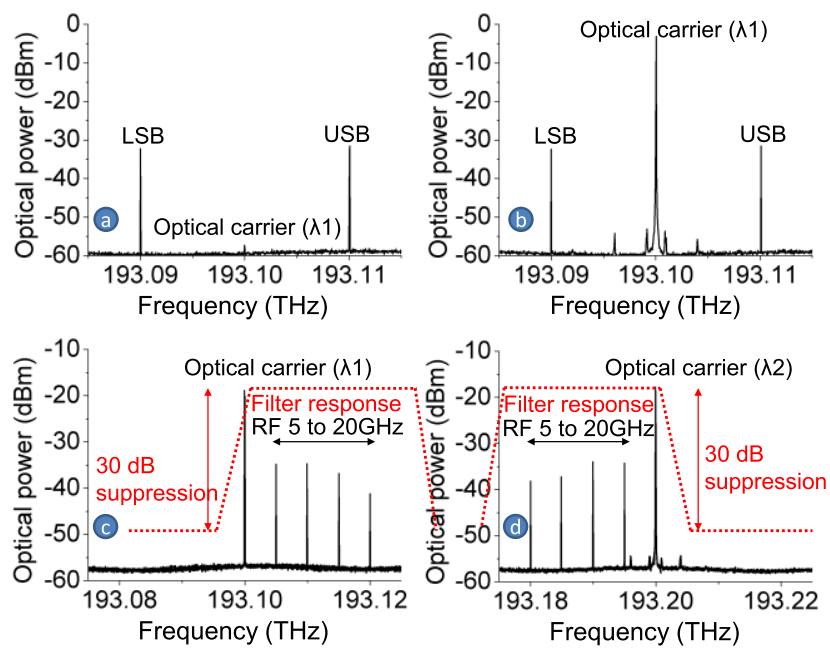

Fig. 3. Optical signal spectra: (a) output of the DPMZM when MZM1 is driven by a RF tone of $10 \mathrm{GHz}$ and both MZM1 and MZM2 are biased at minimum transmission, (b) output of the DPMZM when MZM1 is unchanged but MZM2 biased at maximum transmission, (c) and (d) outputs of the demultiplexer for optical carrier $\lambda_{1}$ and $\lambda_{2}$, respectively, which are modulated by a RF frequency varying between 5 and $20 \mathrm{GHz}$.
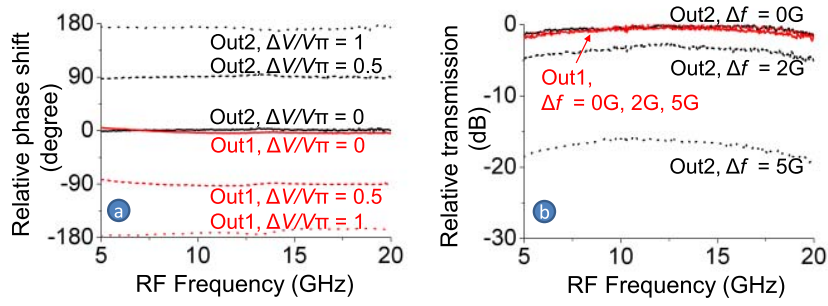

Fig. 4. Demonstration of arbitrary RF splitter functionality: (a) arbitrary phase offsets controlled by a voltage deviation $\Delta V$ on Bias 3 of the DPMZM; (b) arbitrary amplitude offsets controlled by a frequency shift $\Delta f$ of the demultiplexer.

resulting RF outputs 1 and 2, respectively. Figure 4 depicts the $S_{21}$ measurements for a RF bandwidth from 5 to $20 \mathrm{GHz}$. In Fig. 4a arbitrary phase offsets between RF outputs 1 and 2 are demonstrated. Here, the phase offset $\Delta \varphi_{\mathrm{RF}}=-2 \Delta \theta$ is controlled by a voltage deviation $\Delta V$ on Bias 3 of the DPMZM, with the relation $\Delta \theta=\pi \cdot \Delta V / V_{\pi}$ where $V_{\pi}$ is the half-wave voltage of the modulator in terms of the DC bias and has a value of $4 \mathrm{~V}$ in this case. The RF phase reference is defined as being when the detected RF signal of one optical carrier has the same phase shift as that of the other optical carrier. In this experiment, a RF driving power of $-3 \mathrm{dBm}$ was used to implement the small signal modulation as explained in the system principle. However, it is also possible to use a large RF driving power, which will result in significant harmonics in the optical spectrum. In this case, the RF signal bandwidth needs to be reduced to less than an octave, which will allow us to remove the undesired harmonics by means of spectral filtering. Figure $4 \mathrm{~b}$ demonstrates arbitrary amplitude offsets between RF outputs 1 and 2. In this demonstration, the amplitude offset was implemented by downshifting the demultiplexer transmission characteristics (Fig. 3c and 3d) by a frequency deviation $\Delta f$, where optical carrier $\lambda_{2}$ is 

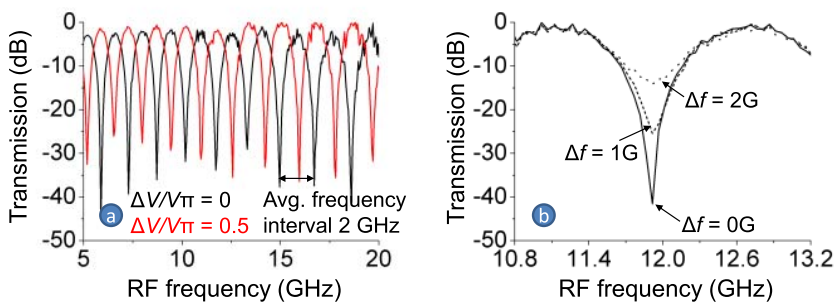

Fig. 5. Demonstration of filter response tuning: (a) frequency shift of the notch positions controlled by a voltage deviation $\Delta V$ on Bias 3 of the DPMZM; (b) notch depth tuning controlled by a frequency shift $\Delta f$ of the demultiplexer.

suppressed as it falls in the transition band while $\lambda_{1}$ stays in the passband and therefore remains unchanged. Although this demonstration is only for the proof-of-concept purpose, the precise control of the amplitude offset can be achieved when a waveguide demultiplexer with a defined transfer function $H(f)$ is used and the frequency alignment of its transition bands to the optical signals is stabilized against the possible drifts caused by the ambient changes or vibrations. Depending on the performance requirement of the applications, a dedicated self-adaptive control subsystem may be incorporated to address the concern of system stability.

\section{Measurements on 2-Tap FIR RF Filter Effect}

Another way to demonstrate the system tunability is to use a filter approach where the two RF outputs are provided with an additional delay difference and are summed together, equivalent to a 2-tap FIR filter [11]. The delay can be introduced either in the electrical domain or in the optical domain. A simple way is to add a dispersion compensating fiber between the DPMZM and demultiplexer. In this case, the expression in (7) can be replaced by

$$
I_{\mathrm{out} 2}=\left(1-\Delta \rho_{\mathrm{RF}}\right) \eta m(t) \cos \left(\omega_{\mathrm{RF}}(t+\Delta \tau)+\Delta \theta\right),
$$

where $\Delta \tau=D L \Delta \lambda$ represents the additional delay of output 2 with respect to output 1 , with $D$ the dispersion coefficient in $\mathrm{ns} / \mathrm{nm} / \mathrm{km}, L$ the length of the fiber, and $\Delta \lambda$ the frequency spacing between the two optical carriers. For simplicity, we assume that the group delay variation within the optical sideband is negligible. In principle, a 2-tap FIR filter is a frequency-periodic notch filter, with the notch interval determined by $\Delta f_{\mathrm{FIR}}=1 / \Delta \tau$; further, the notch positions and the suppression depth can be varied by changing the inter-tap phase and amplitude offset, respectively [11]. This allows us to demonstrate the system tunability by means of varying the notch filter response. Figure 5 depicts the notch filter responses measured when $\Delta \tau=500 \mathrm{ps}$ is used, corresponding to $\Delta f_{\mathrm{FIR}}=2 \mathrm{GHz}$. When we change $\Delta V$ on Bias 3 of the DPMZM, a change of the inter-tap phase offset is achieved, resulting in a frequency shift of the notch positions as demonstrated in Fig. 5a. In Fig. 5b, the tuning of the notch depth is demonstrated, which is achieved when the inter-tap amplitude offset is changed by varying the frequency shift $\Delta f$ of the demultiplexer.

\section{CONCLUSION}

In conclusion, the viability of the proposed MWP system for arbitrary RF splitting was experimentally verified. An instantaneous RF band from 5 to $20 \mathrm{GHz}$ was demonstrated; the bandwidth depends mostly on the bandwidth of the optoelectronic devices in the system. Arbitrary phase and amplitude offsets were achieved by controlling an optical carrier phase shift and the frequency position of the demultiplexer with respect to the optical carriers.

\section{REFERENCES}

[1] J. Capmany and D. Novak, "Microwave photonics combines two worlds," Nature Photon., vol. 1, no. 6, pp. 319-330, 2007.

[2] D. Marpaung, C. Roeloffzen, R. Heideman, A. Leinse, S. Sales, and J. Capmany, "Integrated microwave photonics," Laser Photon. Rev., vol. 7, no. 4, pp. 506-538, Jul. 2013.

[3] S. L. Hahn, Transforms and Applications Handbook. Boca Raton, FL, USA: CRC Press, 2010.

[4] P. Ghelfi et al., "A fully photonics-based coherent radar system," Nature, vol. 501, no. 4792, pp. 341-345, 2014.

[5] L. Zhuang, W. Beeker, A. Leinse, R. Heideman, P. van Dijk, and C. Roeloffzen, "Novel wideband microwave polarization network using a fully-reconfigurable photonic waveguide interleaver with a tworing resonator-assisted asymmetric Mach-Zehnder structure," Opt. Exp., vol. 21, no. 3, pp. 3114-3124, 2013.

[6] T. X. H. Huang, X. Yi, and R. A. Minasian, "Microwave photonic quadrature filter based on an all-optical programmable Hilbert transformer," Opt. Lett., vol. 36, no. 22, pp. 4440-4442, 2011.

[7] W. Liu and J. Yao, "Ultra-wideband microwave photonic phase shifter with a $360^{\circ}$ tunable phase shift based on an erbium-ytterbium co-doped linearly chirped FBG," Opt. Lett., vol. 39, no. 4, pp. 922-924, 2014.

[8] H. Shahoei and J. Yao, "Tunable microwave photonic phase shifter based on slow and fast light effects in a tilted fiber Bragg grating," Opt. Exp., vol. 20, no. 13, pp. 14009-14014, 2012.

[9] H. Chen, M. Sun, Y. Ding, and X. Sun, "Microwave photonic phase shifter based on birefringence effects in a semiconductor optical amplifier," Opt. Lett., vol. 38, no. 17, pp. 3272-3274, 2013.

[10] J. Sancho, J. Lloret, I. Gasulla, S. Sales, and J. Capmany, "Fully tunable $360^{\circ}$ microwave photonic phase shifter based on a single semiconductor optical amplifier," Opt. Exp., vol. 19, no. 18, pp. 17421-17426, 2011.

[11] M. Burla et al., "On-chip CMOS compatible reconfigurable optical delay line with separate carrier tuning for microwave photonic signal processing," Opt. Exp., vol. 19, no. 22, pp. 21475-21484, 2011.

[12] L. Zhuang, M. R. Khan, W. Beeker, A. Leinse, R. Heideman, and C. Roeloffzen, "Novel microwave photonic fractional Hilbert transformer using a ring resonator-based optical all-pass filter," Opt. Exp., vol. 20, no. 24, pp. 26499-26510, 2012.

[13] S. Pan and Y. Zhang, "Tunable and wideband microwave photonic phase shifter based on a single-sideband polarization modulator and a polarizer," Opt. Lett., vol. 37, no. 21, pp. 4483-4485, 2012.

[14] W. Li, W. Zhang, and J. Yao, "A wideband $360^{\circ}$ photonicassisted microwave phase shifter using a polarization modulator and a polarization-maintaining fiber Bragg grating," Opt. Exp., vol. 20, no. 28, pp. 29838-29843, 2012.

[15] X. Sun et al., "Photonic RF phase shifter based on a vectorsum technique using stimulated Brillouin scattering in dispersion shifted fiber," IEEE Trans. Microw. Theory Techn., vol. 58, no. 11, pp. 3206-3212, Nov. 2010.

[16] A. Loayssa and F. J. Lahoz, "Broad-band RF photonic phase shifter based on stimulated Brillouin scattering and single-sideband modulation," IEEE Photon. Technol. Lett., vol. 18, no. 1, pp. 208-210, Jan. 1, 2006.

[17] W. Li et al., "True-time delay line with separate carrier tuning using dual-parallel MZM and stimulated Brillouin scattering-induced slow light," Opt. Exp., vol. 19, no. 13, pp. 12312-12324, 2011.

[18] L. Zhuang, D. Marpaung, M. Burla, W. Beeker, A. Leinse, and C. Roeloffzen, "Low-loss, high-index-contrast $\mathrm{Si}_{3} \mathrm{~N}_{4} / \mathrm{SiO}_{2}$ optical waveguides for optical delay lines in microwave photonics signal processing," Opt. Exp., vol. 19, no. 23, pp. 23162-23170, 2010.

[19] J. H. Baek et al., "10-GHz and 20-GHz channel spacing highresolution AWGs on InP," IEEE Photon. Technol. Lett., vol. 21, no. 15, pp. 298-300, Mar. 1, 2009. 